\title{
A Design of Parameter Optimal Iterative Learning Control for Linear Discrete-Time Systems
}

\author{
Wataru Kase \\ Department of Electrical and Electronic Systems Engineering, Osaka Institute of Technology, 5-16-1 Omiya, Asahi-ku, \\ Osaka 535-8585, Japan \\ Correspondence should be addressed to Wataru Kase; kase@ee.oit.ac.jp
}

Received 9 October 2013; Accepted 5 December 2013; Published 28 January 2014

Academic Editors: S. Biringen, F. Ding, M. Sun, and X.-G. Yan

Copyright (c) 2014 Wataru Kase. This is an open access article distributed under the Creative Commons Attribution License, which permits unrestricted use, distribution, and reproduction in any medium, provided the original work is properly cited.

The iterative learning control algorithm proposed by Owens and Feng, which guarantees the monotonic convergence of the tracking error norms along with the trial, will be modified. The learning gain of the proposed method will be determined through a quadratic cost function. MIMO plant case will be also discussed. Numerical simulations will be presented to confirm the validity of the proposed design.

\section{Introduction}

The iterative learning control (ILC) proposed by Kawamura et al. [1] is a method to control systems operating in a repetitive mode. Examples of such systems include robot manipulator and chemical batch processes reliability testing rigs. The control purpose of ILC is to follow a specified trajectory with high precision. Unlike model matching method [2,3], it might be useful for the plant with nonminimum phase property.

There are many approaches to ILC in the literature [46], for example, the method based on the PD control [1], the inverse systems [7-9], $\mathscr{H}_{\infty}$ control [10,11], and so on. Although the convergence properties of these algorithms have been analyzed, it is not always clear how to choose the free parameters of the algorithms to attain fast or monotonic convergence.

Owens and Feng [12] used parameter optimization through a quadratic cost function as a method to establish the ILC law. The important feature of the algorithm is that the learning gain is to be varied in each trial. The method guarantees the monotonic convergence of tracking error to zero, if a given plant satisfies a definite condition [12]. In the case of nondefinite plants, the behavior of the method was discussed in [13].

In this paper, the method by Owens and Feng will be modified for nondefinite plants. The learning gain is not only for each trial, but also varied at each step. With such modifications, it can be useful for nondefinite plants. Moreover, a special analysis for the tracking error will be shown.

The paper is organized as follows. In the next section, the problem statement will be presented and brief review of the method by Owens and Feng will be given. A derivation of the modified learning gain and error analysis with the proposed gain will be presented in Section 3. A determination of the gain matrix will be shown in Section 4 . The paper was a modified version of the conference paper, and the most different part will be given in Section 5, that is, an extension to the multi-input, multioutput systems. Some simulation results will be given in Section 6 to confirm the effectiveness of the proposed method. Concluding remarks will be given in Section 7.

\section{Problem Statement}

Consider the following $m$-inputs, $m$-outputs linear discretetime system:

$$
\begin{gathered}
x(t+1)=A x(t)+B u(t), \\
y(t)=C x(t),
\end{gathered}
$$

where $u(t), y(t)$, and $x(t)$ are the input, output, and state vector respectively. $t$ is the time instant and $A \in \mathbf{R}^{n \times n}, B \in \mathbf{R}^{n \times m}$, 
and $C \in \mathbf{R}^{m \times n}$. The transfer function $G(z)$ for the above system is given by

$$
G(z)=C(z I-A)^{-1} B
$$

where $z$ is the time-shift operator. Since

$$
(z I-A)^{-1}=z^{-1} I+z^{-2} A+z^{-3} A^{2}+\cdots
$$

the input-output relation of the system (1) can be also described by

$$
y(t)=\sum_{i=1}^{\infty} C A^{i-1} B u(t-i) .
$$

Define the output tracking error $e_{k}(t)$ by

$$
e_{k}(t)=r(t)-y_{k}(t),
$$

where $r(t)$ is the reference signal vector, and the subscript $k$ denotes the trial index; that is, $y_{k}(t)$ means the output vector at time instant $t$ in the $k$ th control trial. $u_{k}(t)$ is defined by the same way. Then, define the column vectors $Y_{k}, U_{k}$, and $E_{k}$ by

$$
\begin{gathered}
Y_{k}=\left[\begin{array}{c}
y_{k}(1) \\
y_{k}(2) \\
\vdots \\
y_{k}(\tau)
\end{array}\right], \quad U_{k}=\left[\begin{array}{c}
u_{k}(0) \\
u_{k}(1) \\
\vdots \\
u_{k}(\tau-1)
\end{array}\right], \\
E_{k}=\left[\begin{array}{c}
e_{k}(1) \\
e_{k}(2) \\
\vdots \\
e_{k}(\tau)
\end{array}\right]=\left[\begin{array}{c}
r(1)-y_{k}(1) \\
r(2)-y_{k}(2) \\
\vdots \\
r(\tau)-y_{k}(\tau)
\end{array}\right] .
\end{gathered}
$$

The purpose of control is to calculate the uniformly bounded input vector $U_{k+1}$ of the form

$$
U_{k+1}=U_{k}+\mathbf{G}_{k} E_{k}
$$

which makes $E_{k} \rightarrow 0$ as $k \rightarrow \infty$, where $\mathbf{G}_{k}$ is a gain matrix to be determined $\left(\mathbf{G}_{1}=I\right)$.

Temporarily, it is assumed that $m=1$ that is, we will consider the single-input, single-output case. Owens and Feng [12] considered the case where $\mathbf{G}_{k}=g_{k} I$ ( $g_{k}$ is a scalar). Then,

$$
\begin{aligned}
Y_{k+1} & =T_{\tau-1}\left(U_{k}+g_{k} E_{k}\right) \\
& =Y_{k}+g_{k} \mathbf{T}_{\tau-1} E_{k},
\end{aligned}
$$

where

$$
\mathbf{T}_{\tau-1}=\left[\begin{array}{cccc}
C B & 0 & \cdots & 0 \\
C A B & C B & \cdots & 0 \\
\vdots & \vdots & \ddots & \vdots \\
C A^{\tau-1} B & C A^{\tau-2} B & \cdots & C B
\end{array}\right] .
$$

Set the cost function $J\left(g_{k}\right)$ by

$$
\begin{aligned}
J\left(g_{k}\right)= & \left\|E_{k+1}\right\|^{2}+\rho g_{k}^{2}, \quad \rho>0 \\
= & \left\|\left(I-g_{k} \mathbf{T}_{\tau-1}\right) E_{k}\right\|^{2}+\rho g_{k}^{2} \\
= & \left(\rho+\left\|\mathbf{T}_{\tau-1} E_{k}\right\|^{2}\right) g_{k}^{2}-2 E_{k}^{T} \mathbf{T}_{\tau-1} E_{k} g_{k} \\
& +\left\|E_{k}\right\|^{2} .
\end{aligned}
$$

The value $g_{k}$ which minimizes the above cost is given by

$$
g_{k}=\frac{E_{k}^{T} \mathbf{T}_{\tau-1} E_{k}}{\rho+\left\|\mathbf{T}_{\tau-1} E_{k}\right\|^{2}} .
$$

Then,

$$
\begin{aligned}
& E_{k+1}^{T} E_{k+1}-E_{k}^{T} E_{k} \\
& \quad=E_{k}^{T}\left(I-g_{k} \mathbf{T}_{\tau-1}^{T}\right)\left(I-g_{k} \mathbf{T}_{\tau-1}\right) E_{k}-E_{k}^{T} E_{k} \\
& \quad=E_{k}^{T}\left\{g_{k}^{2} \mathbf{T}_{\tau-1}^{T} \mathbf{T}_{\tau-1}-g_{k}\left(\mathbf{T}_{\tau-1}^{T}+\mathbf{T}_{\tau-1}\right)\right\} E_{k} \\
& \quad=g_{k}^{2}\left\{\left\|\mathbf{T}_{\tau-1} E_{k}\right\|^{2}-2\left(\rho+\left\|\mathbf{T}_{\tau-1} E_{k}\right\|^{2}\right)\right\} \\
& \quad=-g_{k}^{2}\left(2 \rho+\left\|\mathbf{T}_{\tau-1} E_{k}\right\|^{2}\right) \leq 0,
\end{aligned}
$$

and thus $E_{k}^{T} E_{k} \geq 0$ converges to a nonnegative value. Since $2 \rho+\left\|\mathbf{T}_{\tau-1} E_{k}\right\|^{2}>0$,

$$
\lim _{k \rightarrow \infty} g_{k}=\lim _{k \rightarrow \infty} E_{k}^{T} \mathbf{T}_{\tau-1} E_{k}=0 .
$$

Therefore, $E_{k} \rightarrow 0$ if $\mathbf{T}_{\tau-1}^{T}+\mathbf{T}_{\tau-1}>0$ or $\mathbf{T}_{\tau-1}^{T}+\mathbf{T}_{\tau-1}<0$. In the following sections, the gain which makes $E_{k} \rightarrow 0$ without the above definite conditions will be derived.

\section{Derivation of Diagonal Gain Matrix}

Applying the control input (7) to (8),

$$
\begin{gathered}
Y_{k+1}=\mathbf{T}_{\tau-1} U_{k+1} \\
=\mathbf{T}_{\tau-1}\left(U_{k}+\mathbf{G}_{k} E_{k}\right) \\
=Y_{k}+\mathbf{T}_{\tau-1} \overline{\mathbf{E}}_{k} \bar{G}_{k}, \\
\overline{\mathbf{E}}_{k}:=\left[\begin{array}{ccc}
e_{k}(1) & \cdots & 0 \\
\vdots & \ddots & \vdots \\
0 & \cdots & e_{k}(\tau)
\end{array}\right], \quad \bar{G}_{k}:=\left[\begin{array}{c}
g_{k}(1) \\
\vdots \\
g_{k}(\tau)
\end{array}\right] .
\end{gathered}
$$

Therefore,

$$
E_{k+1}=E_{k}-\mathbf{T}_{\tau-1} \overline{\mathbf{E}}_{k} \bar{G}_{k} .
$$

Set cost function $J\left(\bar{G}_{k}\right)$ by

$$
J\left(\bar{G}_{k}\right)=\left\|E_{k+1}\right\|^{2}+\rho\left\|\bar{G}_{k}\right\|^{2}, \quad \rho>0 .
$$

Then, using (15),

$$
\begin{aligned}
J\left(\bar{G}_{k}\right)= & \left\|E_{k}-\mathbf{T}_{\tau-1} \overline{\mathbf{E}}_{k} \bar{G}_{k}\right\|^{2}+\rho\left\|\bar{G}_{k}\right\|^{2} \\
= & \bar{G}_{k}^{T} \Gamma_{k} \bar{G}_{k}-\bar{G}_{k}^{T} \overline{\mathbf{E}}_{k}^{T} \mathbf{T}_{\tau-1}^{T} E_{k} \\
& -E_{k}^{T} \mathbf{T}_{\tau-1} \overline{\mathbf{E}}_{k} \bar{G}_{k}+\left\|E_{k}\right\|^{2} \\
= & \left\|\Gamma_{k}^{1 / 2} \bar{G}_{k}-\Gamma_{k}^{-1 / 2} \overline{\mathbf{E}}_{k}^{T} \mathbf{T}_{\tau-1}^{T} E_{k}\right\|^{2} \\
& +\left\|E_{k}\right\|^{2}-\left\|\Gamma_{k}^{-1 / 2} \overline{\mathbf{E}}_{k}^{T} \mathbf{T}_{\tau-1}^{T} E_{k}\right\|^{2},
\end{aligned}
$$


where

$$
\Gamma_{k}:=\rho I+\overline{\mathbf{E}}_{k}^{T} \mathbf{T}_{\tau-1}^{T} \mathbf{T}_{\tau-1} \bar{E}_{k} .
$$

Therefore, $J\left(\bar{G}_{k}\right)$ is minimized if

$$
\begin{aligned}
\bar{G}_{k} & =\Gamma_{k}^{-1} \overline{\mathbf{E}}_{k}^{T} \mathbf{T}_{\tau-1}^{T} E_{k} \\
& =\overline{\mathbf{E}}_{k}^{T} \mathbf{T}_{\tau-1}^{T}\left(\rho I+\mathbf{T}_{\tau-1} \overline{\mathbf{E}}_{k} \overline{\mathbf{E}}_{k}^{T} \mathbf{T}_{\tau-1}^{t}\right)^{-1} E_{k} .
\end{aligned}
$$

Substituting (18) to (15),

$$
E_{k+1}=\left(I-\mathbf{T}_{\tau-1} \overline{\mathbf{E}}_{k} \Gamma_{k}^{-1} \overline{\mathbf{E}}_{k}^{T} \mathbf{T}_{\tau-1}^{T}\right) E_{k}
$$

Consider the following candidate of Lyapunov function $V_{k}$ :

$$
V_{k}:=E_{k}^{T} E_{k} \geq 0 \text {. }
$$

Then,

$$
\begin{aligned}
\Delta V_{k+1}:= & V_{k+1}-V_{k} \\
= & E_{k}^{T}\left\{\left(I-\mathbf{T}_{\tau-1} \overline{\mathbf{E}}_{k} \Gamma_{k}^{-1} \overline{\mathbf{E}}_{k}^{T} \mathbf{T}_{\tau-1}^{T}\right)^{2}-I\right\} E_{k} \\
= & E_{k}^{T}\left\{\left(\mathbf{T}_{\tau-1} \overline{\mathbf{E}}_{k} \Gamma_{k}^{-1} \overline{\mathbf{E}}_{k}^{T} \mathbf{T}_{\tau-1}^{T}\right)^{2}\right. \\
& \left.\quad-2 \mathbf{T}_{\tau-1} \overline{\mathbf{E}}_{k} \Gamma_{k}^{-1} \overline{\mathbf{E}}_{k}^{T} \mathbf{T}_{\tau-1}^{T}\right\} E_{k} \\
= & \bar{G}_{k}^{T}\left(\overline{\mathbf{E}}_{k}^{T} \mathbf{T}_{\tau-1}^{T} \mathbf{T}_{\tau-1} \overline{\mathbf{E}}_{k}-2 \Gamma_{k}\right) \bar{G}_{k} \\
= & -\bar{G}_{k}^{T}\left(2 \rho I+\overline{\mathbf{E}}_{k}^{T} \mathbf{T}_{\tau-1}^{T} \mathbf{T}_{\tau-1} \overline{\mathbf{E}}_{k}\right) \bar{G}_{k} \\
\leq & 0 .
\end{aligned}
$$

Therefore, $V_{k}$ becomes the Lyapunov function and $\Delta V_{k} \rightarrow 0$ as $k \rightarrow \infty$. Since

$$
2 \rho I+\overline{\mathbf{E}}_{k}^{T} \mathbf{T}_{\tau-1}^{T} \mathbf{T}_{\tau-1} \overline{\mathbf{E}}_{k}>0
$$

it follows that

$$
\lim _{k \rightarrow \infty} \bar{G}_{k}=\lim _{k \rightarrow \infty} \overline{\mathbf{E}}_{k}^{T} \mathbf{T}_{\tau-1}^{T} E_{k}=0 .
$$

Hence

$$
\begin{aligned}
& \overline{\mathbf{E}}_{k}^{T} \mathbf{T}_{\tau-1}^{T} E_{k} \\
& =\left[\begin{array}{ccc}
e_{k}(1) & \cdots & 0 \\
\vdots & \ddots & \vdots \\
0 & \cdots & e_{k}(\tau)
\end{array}\right]\left[\begin{array}{ccc}
C B & \cdots & C A^{\tau-1} B \\
\vdots & \ddots & \vdots \\
0 & \cdots & C B
\end{array}\right]\left[\begin{array}{c}
e_{k}(1) \\
e_{k}(2) \\
\vdots \\
e_{k}(\tau)
\end{array}\right] \\
& =\left[\begin{array}{c}
C B e_{k}^{2}(1)+e_{k}(1) \sum_{i=2}^{\tau} C A^{i-1} B e_{k}(i) \\
\vdots \\
C B e_{k}^{2}(\tau-2)+e_{k}(\tau-2) \sum_{i=\tau-1}^{\tau} C A^{i-\tau+2} B e_{k}(i) \\
C B e_{k}^{2}(\tau-1)+C A B e_{k}(\tau) e_{k}(\tau-1) \\
C B e_{k}^{2}(\tau)
\end{array}\right] .
\end{aligned}
$$

From the bottom element of the above vector, it follows that

$$
\lim _{k \rightarrow \infty} e_{k}(\tau)=0
$$

if $C B \neq 0$. Substituting the above relation to (25), it follows that $\overline{\mathbf{E}}_{k}^{T} \mathbf{T}_{\tau-1}^{T} E_{k}$

$$
=\left[\begin{array}{c}
C B e_{k}^{2}(1)+e_{k}(1) \sum_{i=2}^{\tau-1} C A^{i-1} B e_{k}(i) \\
\vdots \\
C B e_{k}^{2}(\tau-2)+C A B e_{k}(\tau-2) e_{k}(\tau-1) e_{k}(\tau-2) \\
C B e_{k}^{2}(\tau-1) \\
0
\end{array}\right]
$$

and thus

$$
\lim _{k \rightarrow \infty} e_{k}(\tau-1)=0
$$

and so on. Therefore,

$$
\lim _{k \rightarrow \infty} E_{k}(\tau)=\lim _{k \rightarrow \infty}\left[\begin{array}{c}
e_{k}(1) \\
e_{k}(2) \\
\vdots \\
e_{k}(\tau)
\end{array}\right]=0 .
$$

Even if $C B=0$, there exists the integer $d$ such that $C B=\cdots=C A^{d-1} B=0$ and $C A^{d} B \neq 0$, and thus the above discussion is not loss of generality.

\section{Nondiagonal Gain Matrix}

Define the following matrices:

$$
\overline{\mathbf{E}}_{k}:=\left[\begin{array}{ccc}
E_{k}^{T} & \cdots & 0 \\
\vdots & \ddots & \vdots \\
0 & \cdots & E_{k}^{T}
\end{array}\right], \quad \bar{G}_{k}=\operatorname{rs}\left[\mathbf{G}_{k}\right],
$$

where $\mathrm{rs}[\cdot]$ denotes the row string of matrix. Then,

$$
\mathbf{G}_{k} E_{k}=\overline{\mathbf{E}}_{k} \bar{G}_{k}
$$

holds, and the discussions in the previous section also hold. Moreover,

$$
\begin{aligned}
\overline{\mathbf{E}}_{k}^{T} \bar{T}_{\tau-1}^{T} E_{k} & \\
= & {\left[\begin{array}{ccc}
E_{k} & \cdots & 0 \\
\vdots & \ddots & \vdots \\
0 & \cdots & E_{k}^{T}
\end{array}\right]\left[\begin{array}{ccc}
C B & \cdots & C A^{\tau-1} B \\
\vdots & \ddots & \vdots \\
0 & \cdots & C B
\end{array}\right]\left[\begin{array}{c}
e_{k}(1) \\
e_{k}(2) \\
\vdots \\
e_{k}(\tau)
\end{array}\right] } \\
= & {\left[\begin{array}{c}
\sum_{i=1}^{\tau} C A^{i-1} B E_{k} e_{k}(i) \\
\vdots \\
\sum_{i=\tau-2}^{\tau} C A^{i-\tau+2} B E_{k} e_{k}(i) \\
C B E_{k} e_{k}(\tau-1)+C A B E_{k} e_{k}(\tau) \\
C B E_{k} e_{k}(\tau)
\end{array}\right] \longrightarrow 0 . }
\end{aligned}
$$


If $C B \neq 0$, it follows that

$$
\lim _{k \rightarrow \infty} E_{k} e_{k}(\tau)=\lim _{k \rightarrow \infty}\left[\begin{array}{c}
e_{k}(1) e_{k}(\tau) \\
e_{k}(2) e_{k}(\tau) \\
\vdots \\
e_{k}^{2}(\tau)
\end{array}\right]
$$

from the bottom element in (32), and thus

$$
\lim _{k \rightarrow \infty} e_{k}(\tau)=0
$$

Substituting the above relation to (32), it follows that

$$
\begin{aligned}
& \lim _{k \rightarrow \infty} \overline{\mathbf{E}}_{k}^{T} \bar{T}_{\tau-1}^{T} E_{k} \\
& =\left[\begin{array}{c}
\tau-1 \\
\sum_{i=1} C A^{i-1} B E_{k} e_{k}(i) \\
\vdots \\
C B E_{k} e_{k}(\tau-2)+C A B E_{k} e_{k}(\tau-1) \\
C B E_{k} e_{k}(\tau-1) \\
0
\end{array}\right]
\end{aligned}
$$

and thus $\lim _{k \rightarrow \infty} e_{k}(\tau-1)$ and so on. Therefore,

$$
\lim _{k \rightarrow \infty} E_{k}=\lim _{k \rightarrow \infty}\left[\begin{array}{c}
e_{k}(1) \\
e_{k}(2) \\
\vdots \\
e_{k}(\tau)
\end{array}\right]=0 .
$$

As is in the previous discussions, it is not loss of generality if $C B=0$.

It is worth noting that the expression of the second equality of (19) for $\bar{G}_{k}$ is powerful. The expression needs the inversion of $\tau \times \tau$ matrix, while the first expression needs the inversion of $\tau^{2} \times \tau^{2}$.

\section{Multi-Input, Multioutput Plant}

Although the almost same analysis of the output error holds for the multi-input, multioutput plant, it needs the nonsingularity of $C B$. For this problem, consider a polynomial matrix $L(z)$ satisfying

$$
\lim _{z \rightarrow \infty} L(z) G(z)=K \quad \text { (nonsingular). }
$$

Such $L(z)$ is called an interactor matrix for $G(z)$ [14]. For easiness, we assume that $K=I_{m}$. It is reported that the coefficient matrix of $L(z)$ satisfies the following relation [15]:

$$
\mathbf{L T}_{w-1}=\mathbf{J}_{w-1},
$$

where

$$
\begin{gathered}
L(z)=z L_{1}+z^{2} L_{2}+\cdots+z^{w} L_{w}, \\
\mathbf{L}=\left[\begin{array}{lll}
L_{1} & \cdots & L_{w}
\end{array}\right], \quad L_{i} \in \mathbf{R}^{m \times m}, \\
\mathbf{J}_{w-1}=\left[\begin{array}{ll}
I_{m} & 0_{m \times m(w-1)}
\end{array}\right]
\end{gathered}
$$

and $w$ is the least integer which satisfies

$$
\operatorname{rank}\left[\begin{array}{c}
\mathbf{T}_{w-1} \\
\mathbf{J}_{w-1}
\end{array}\right]=\operatorname{rank} \mathbf{T}_{w-1}
$$

Equation (38) is solvable from the above condition, and $\mathbf{L}$ is given by

$$
\mathbf{L}=\mathbf{J}_{w-1} \mathbf{T}_{w-1}^{\dagger}
$$

using Moore-Penrose pseudoinverse $\mathbf{T}_{w-1}^{\dagger}$ of $\mathbf{T}_{w-1}$. It is reported that all zeros of $\operatorname{det} L(z)$ lie at the origin if $\mathrm{L}$ is given by (41) [3].

Define the feedback gain $\widetilde{C}$ by

$$
\widetilde{C}:=\mathbf{L}\left[\begin{array}{c}
C \\
C A \\
\vdots \\
C A^{w-1}
\end{array}\right] .
$$

Then, it is clear that

$$
\widetilde{C} B=I_{m}
$$

For the compensated plant $\widetilde{C}(z I-A)^{-1} B=z^{-1} L(z) G(z)$, design the ILC system presented in the previous section. That is, define the compensated reference signal and output vector by

$$
\begin{aligned}
\widetilde{r}(t) & =z^{-1} L(z) r(t), \\
\tilde{y}_{k}(t) & =z^{-1} L(z) y_{k}(t) \\
& =z^{-1} L(z) G(z) u_{k}(t) .
\end{aligned}
$$

Then, the augmented tracking error $\widetilde{e}_{k}(t)$ is given by

$$
\lim _{k \rightarrow \infty} \widetilde{e}_{k}(t)=0
$$

On the other hand, the relation between $e_{k}(t)$ and $\widetilde{e}_{k}(t)$ is described by

$$
e_{k}(t)=z L^{-1}(z) \widetilde{e}_{k}(t)
$$

Since $\operatorname{det} z L^{-1}(z)$ is stable [3], it yields that

$$
\lim _{k \rightarrow \infty} e_{k}(t)=0
$$

\section{Numerical Examples}

Set $(A, B, C)$ in (1) by

$$
\begin{gathered}
A=\left[\begin{array}{cc}
0 & 1 \\
-0.5 & -1
\end{array}\right], \quad B=\left[\begin{array}{l}
0 \\
1
\end{array}\right], \\
C=\left[\begin{array}{ll}
1.2 & 1
\end{array}\right] .
\end{gathered}
$$

In this case, the control objective is nonminimum phase plant and the definite conditions are not satisfied. 


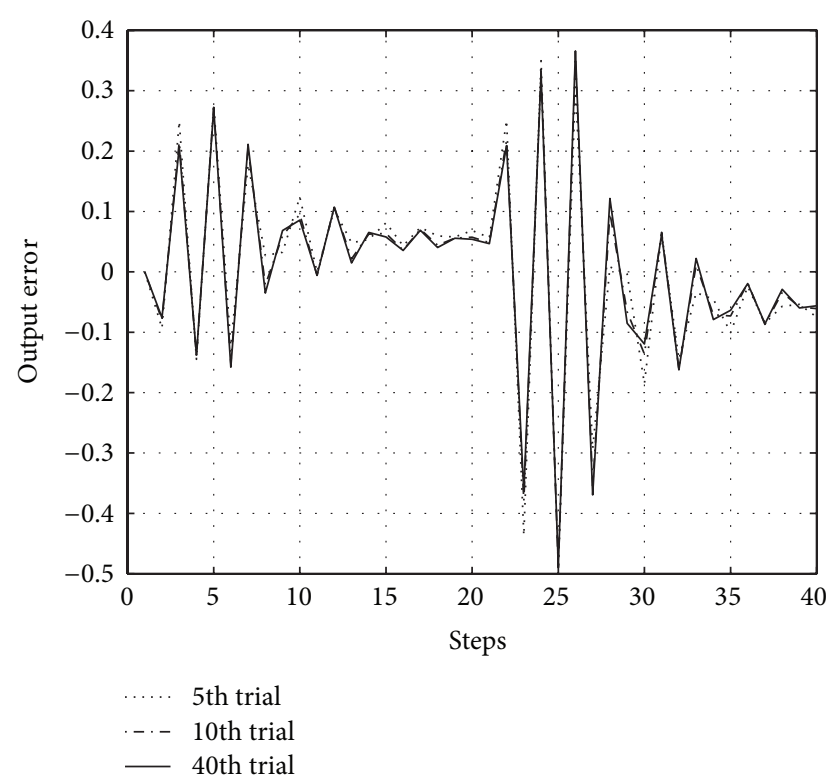

FIGURE 1: Output tracking error with scalar gain.

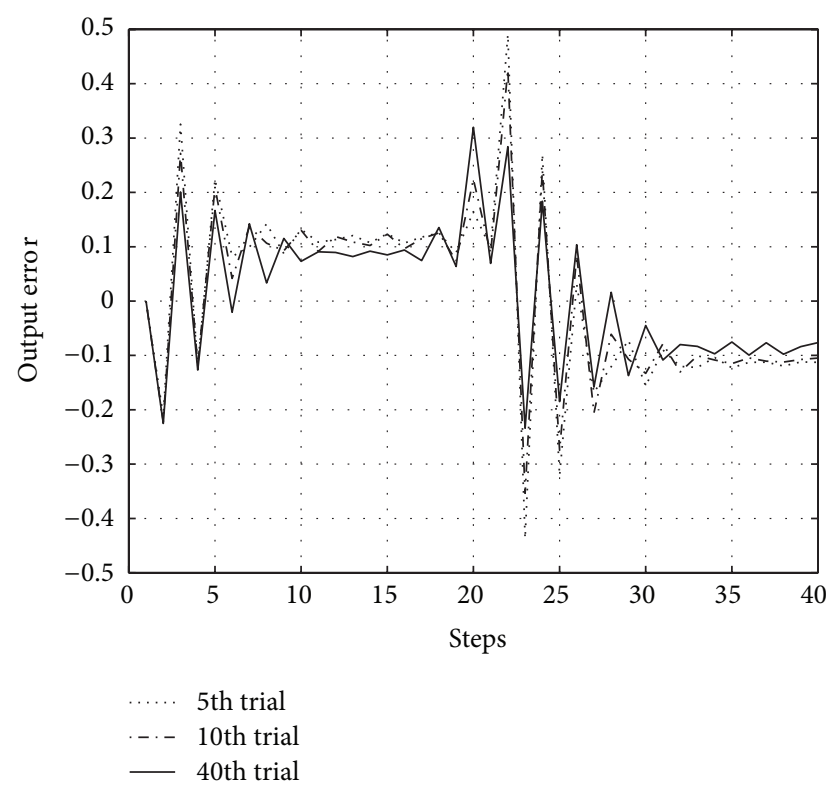

FIGURE 2: Output tracking error with diagonal gain matrix.

Reference signal $r(t)$ is given by

$$
r(t)= \begin{cases}1 & (1 \leq t \leq 20) \\ -1 & (t \geq 21)\end{cases}
$$

and $\rho=1$. Figure 1 shows the output tracking errors of scalar gain ILC proposed by Owens and Feng, where errors of the 5 th, 10th, and 40th trial are shown. Since the learning does not proceed, it is hard to distinguish these three lines.

Figure 2 shows the output tracking errors of the diagonal matrix gain proposed in Section 3, where errors of the 5th, 10 th, and 40 th trial are shown. It can be seen that the errors decrease monotonically. Comparing Figures 1 and 2, it seems

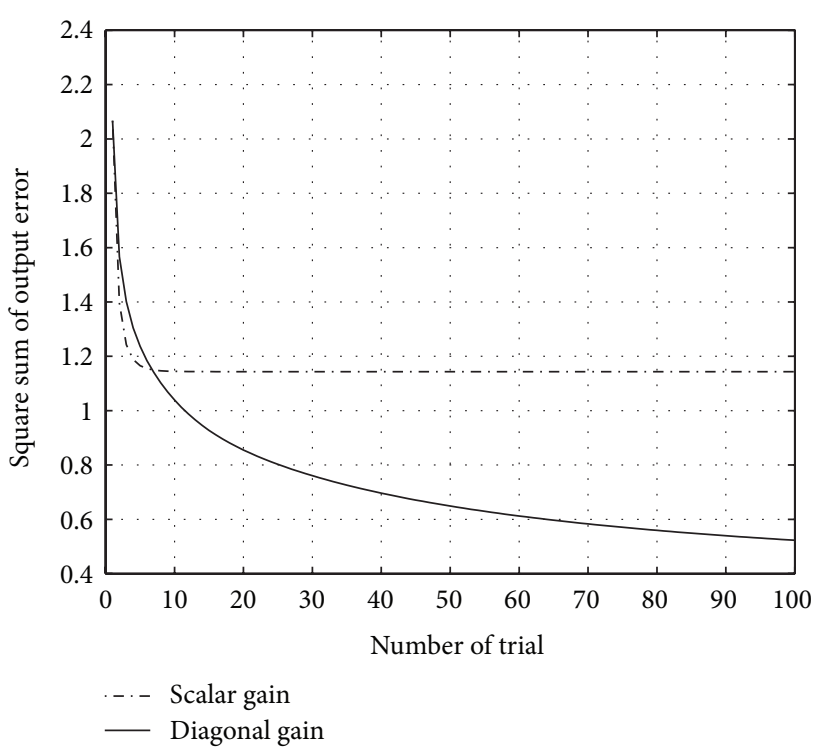

FIGURE 3: Square sum of output tracking errors.

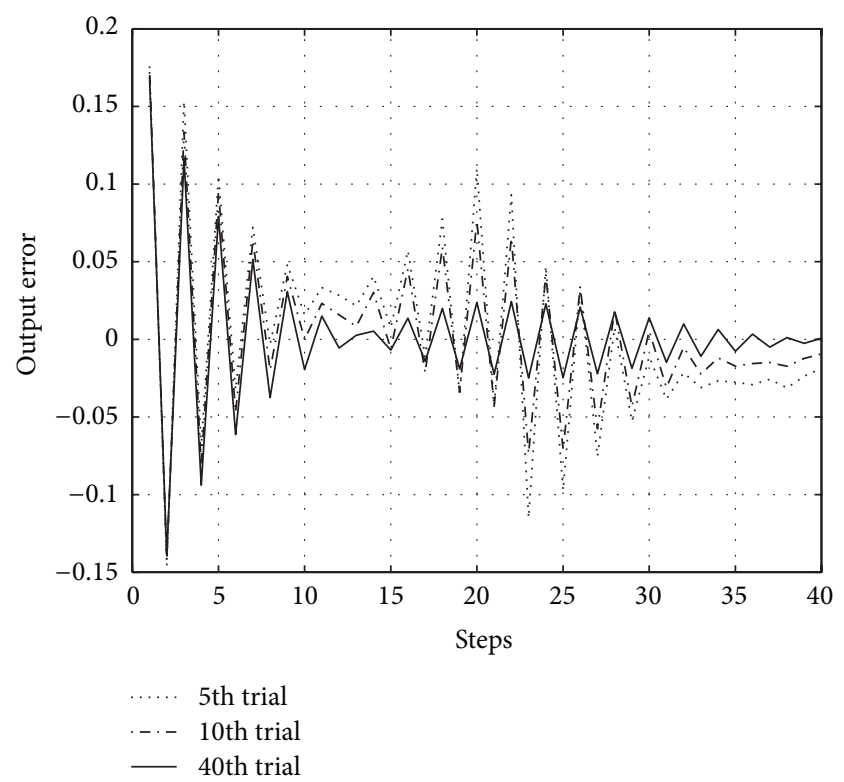

FIgURE 4: Output tracking error with nondiagonal gain matrix.

that better trackings are achieved partially by the scalar gain (Figure 1) than the matrix gain. Figure 3 shows the relations between the square sum of output tracking errors and the number of trials. By the scalar gain, the learning is stopped till 10th trial, whereas the sums are decreased monotonically by the matrix gain. Figure 4 shows the result by the method presented in Section 4 . The result seems much better than the results in Figures 1 and 2. From these points, the effectiveness of the proposed ILC was confirmed.

\section{Conclusion}

In this paper, the method proposed by Owens and Feng [12] was modified, and the assumption to confirm the tracking 
errorconvergence to zero was removed. It was extended to the multi-input, multioutput case. Simulation results were shown for validity of the proposed method.

In this paper, it was only considered the ideal case. It should be discussed for the plant having uncertain or timevarying parameters and nonlinearity. It will also considered the feedback control of the proposed method.

\section{Conflict of Interests}

The author declares that there is no conflict of interests regarding the publication of this paper.

\section{References}

[1] S. Kawamura, F. Miyazaki, and S. Arimoto, "Bettering operation of robots by learning," IEEE Transactions on Systems, Man and Cybernetics, vol. 1, pp. 123-140, 1984.

[2] W. A. Wolovich, Linear Multivariable Systems, Springer, Berlin, Germany, 1974.

[3] W. Kase and Y. Mutoh, "Suboptimal exact model matching for multivariable systems with measurement noise," IEEE Transactions on Automatic Control, vol. 45, no. 6, pp. 1170-1175, 2000.

[4] K. Moore, Iterative Learning Control for Deterministic Systems, Springer, Berlin, Germany, 1993.

[5] D. A. Bristow, M. Tharayil, and A. G. Alleyne, "A survey of iterative learning control: a learning-based method for highperformance tracking control," IEEE Control Systems Magazine, vol. 26, no. 3, pp. 96-114, 2006.

[6] K. L. Moore and X. U. Jian-Xin, "Special issue on iterative learning control," International Journal of Control, vol. 73, no. 10, pp. 819-823, 2000.

[7] K. Furuta and M. Yamakita, "The design of a learning control systems for multivariable systems," in Proceedings of the IEEE International Symposium on Intelligent Control, pp. 371-376, 1987.

[8] J. Ghosh and B. Paden, "Pseudo-inverse based iterative learning control for nonlinear plants with disturbances," in Proceedings of the 38th IEEE Conference on Decision and Control (CDC '99), pp. 5206-5212, December 1999.

[9] K. Kinosita, T. Sogo, and N. Adachi, "Iterative learning control using adjoint systems and stable inversion," Asian Journal of Control, vol. 4, no. 1, pp. 60-67, 2002.

[10] C. J. Goh and W. Y. Van, "An $\mathscr{H}_{\infty}$ synthesis of robust current error feedback learning control," Journal of Dynamic Systems, Measurement and Control, Transactions of the ASME, vol. 118, no. 2, pp. 341-346, 1996.

[11] D. de Roover and O. H. Bosgra, "Synthesis of robust multivariable iterative learning controllers with application to a wafer stage motion system," International Journal of Control, vol. 73, no. 10, pp. 968-979, 2000.

[12] D. H. Owens and K. Feng, "Parameter optimization in iterative learning control," International Journal of Control, vol. 76, no. 11, pp. 1059-1069, 2003.

[13] D. H. Owens, M. Tomas-Rodriguez, and J. J. Hatonen, "Limiting behaviour in parameter optimal iterative learning control," International Journal of Automation and Computing, vol. 3, pp. 222-228, 2006.

[14] W. A. Wolovich and P. L. Falb, "Invariants and canonical forms under dynamic compensation," SIAM Journal on Control and Optimization, vol. 14, no. 6, pp. 996-1008, 1976.
[15] Y. Mutoh and R. Ortega, "Interactor structure estimation for adaptive control of discrete-time multivariable nondecouplable systems," Automatica, vol. 29, no. 3, pp. 635-647, 1993. 


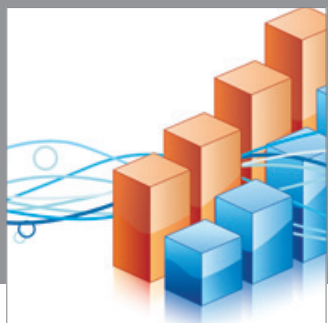

Advances in

Operations Research

mansans

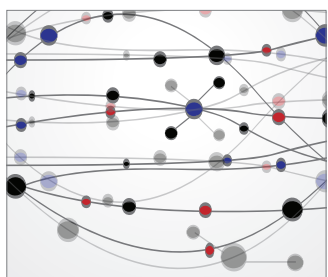

The Scientific World Journal
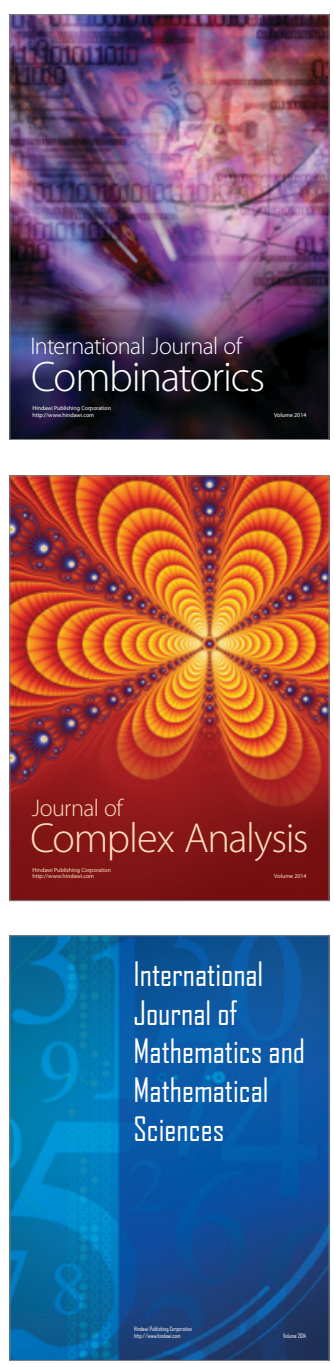
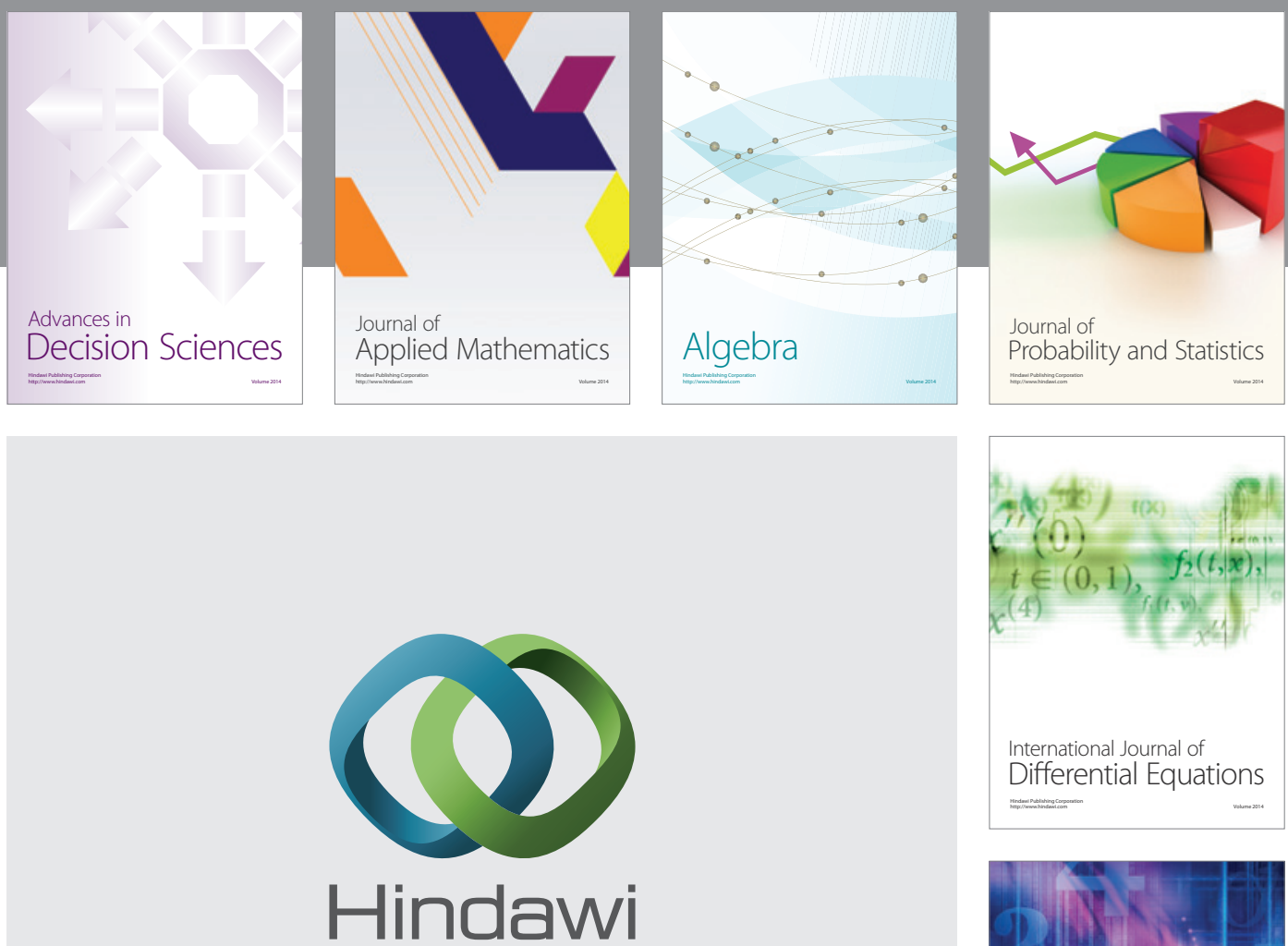

Submit your manuscripts at http://www.hindawi.com
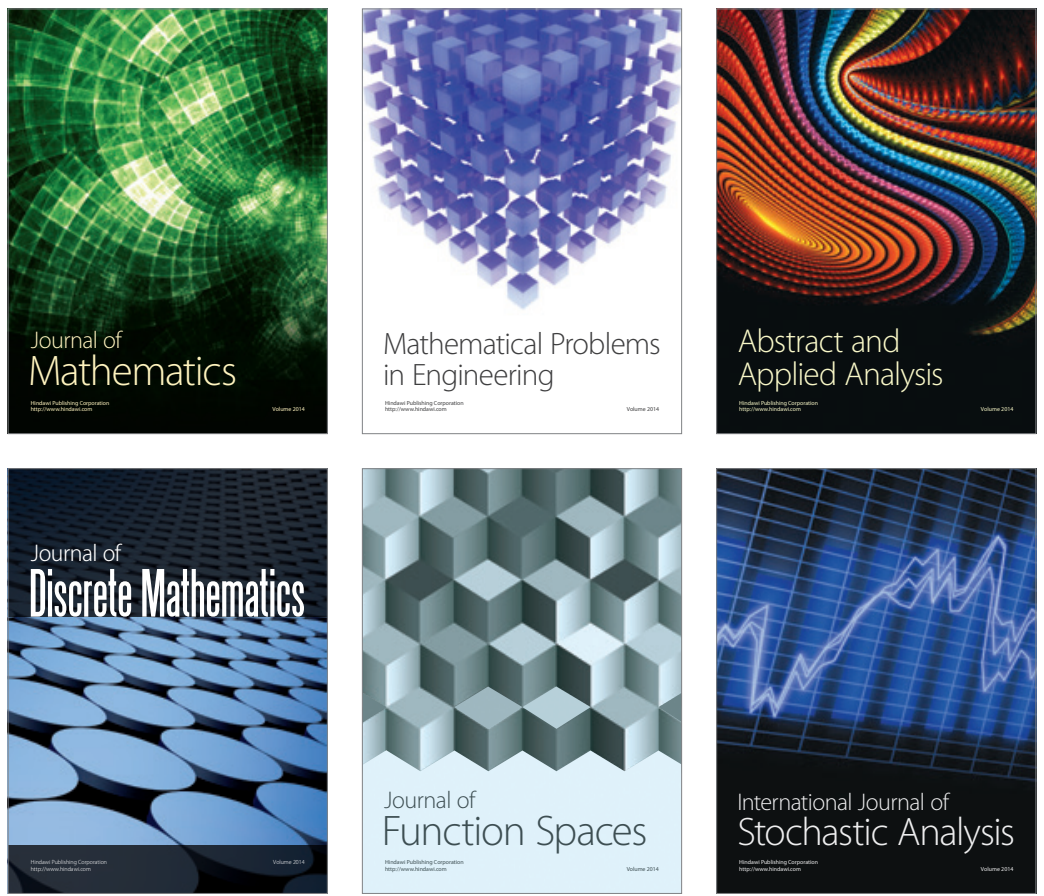

Journal of

Function Spaces

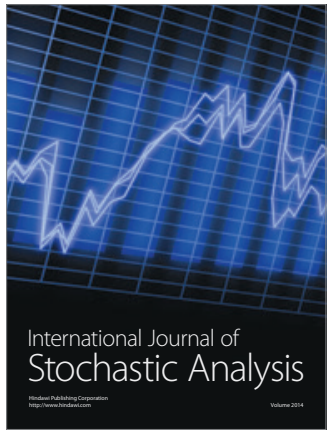

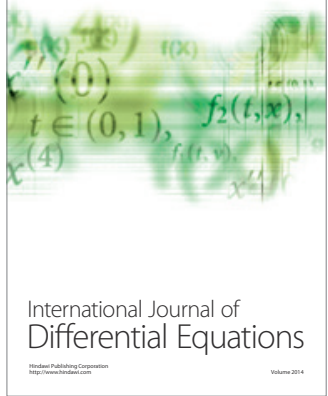
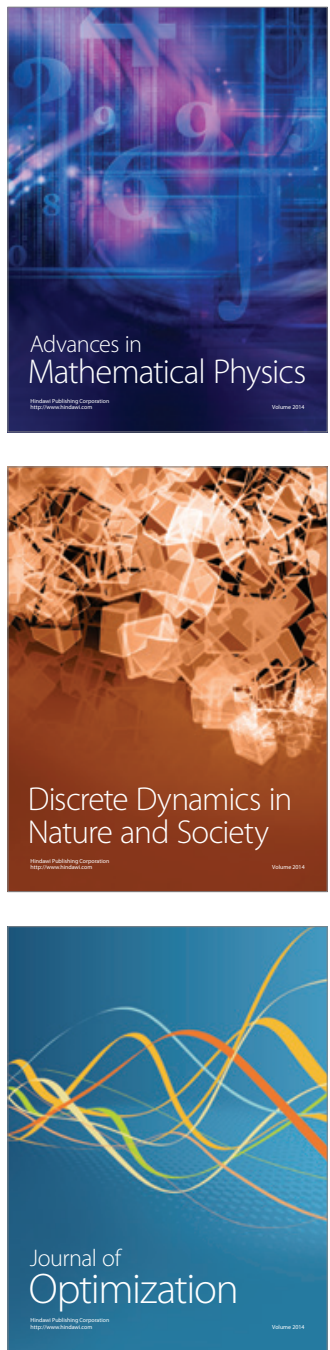